\title{
A Process Evaluation of an Environmental Intervention: The Case of a Heritage Tourism Organization: An Abstract
}

\author{
Diana Gregory-Smith, Victoria K. Wells, Danae Manika, and David McElroy
}

\begin{abstract}
Using a case study methodology, this paper assesses an environmental intervention among employees of a cultural heritage tourism organization. The intervention took a downstream social marketing approach and focused on influencing individual employee behaviors within the heritage organization, such as energy saving (i.e., lighting and heating) and recycling (i.e., waste reduction). It was delivered via a customizable "sustainability toolkit." The assessment of the intervention process is based on data collected from the heritage organization's employees and the reflections of sustainability practitioners (external to the organization) who designed and delivered the intervention. More specifically, using Pawson and Tilley's (1997) context-mechanism-outcome framework, we carry out an evaluation of the environmental intervention, and we uncover that a good understanding of the tourism and organizational context (regarding the dimensions of structure, culture, agency, and relations) and the use of tailored, action-focused mechanisms (for each context dimension) are critical to achieving transformational outcomes in environmental interventions within cultural heritage organizations. Implications for future interventions and for tourism management and practice are discussed along with further research directions.
\end{abstract}

\footnotetext{
D. Gregory-Smith $(\triangle)$

University of Birmingham, Birmingham, UK

e-mail: d.gregory-smith@bham.ac.uk

V.K. Wells

University of Sheffield, Sheffield, UK

e-mail: victoria.wells@sheffield.ac.uk

D. Manika

Mary University of London, London, UK

e-mail: d.manika@qmul.ac.uk

D. McElroy

Global Action Plan, London, UK

e-mail: david.mcelroy@globalactionplan.org.uk
} 